\title{
Ground States for Nonlocal Schrödinger Type Operators on Locally Compact Abelian Groups
}

\author{
Anatoly N. Kochubei \\ Institute of Mathematics, \\ National Academy of Sciences of Ukraine, \\ Tereshchenkivska 3, \\ Kyiv, 01004 Ukraine \\ Email: kochubei@imath.kiev.ua \\ Yuri Kondratiev \\ Department of Mathematics, University of Bielefeld, \\ D-33615 Bielefeld, Germany, \\ Email: kondrat@math.uni-bielefeld.de
}

\begin{abstract}
We find classes of nonlocal operators of Schrödinger type on a locally compact noncompact Abelian group $\mathfrak{G}$, for which there exists a ground state. In particular, such a result is obtained for the case where the principal part of our operator generates a recurrent random walk. Explicit conditions for the existence of a ground state are obtained for the case $\mathfrak{G}=\mathbb{Q}_{p}^{n}$ where $\mathbb{Q}_{p}$ is the field of $p$-adic numbers.
\end{abstract}

Key words: ground state; locally compact Abelian group; operators of Schrödinger type; recurrent random walk; field of $p$-adic numbers

MSC 2010. Primary: 47G10. Secondary: 45P05; 11S80, 43A70. 


\section{Introduction}

In a number of applications (see the references in [10]) we encounter nonlocal Schrödinger operators

$$
(\mathcal{L} u)(x)=-m(x) u(x)+\int_{\mathbb{R}^{n}} a(x-y) u(y) d y
$$

where $a(x) \geqslant 0$ is an even bounded continuous function, such that $\int_{\mathbb{R}^{n}} a(x) d x=1$, and typically $m \in C_{b}\left(\mathbb{R}^{n}\right), 0 \leqslant m(x) \leqslant 1, m(x) \rightarrow 1$ as $|x| \rightarrow \infty$. The recent papers [ㅇ, 9, 10] contain a rather well-developed spectral theory of such operators, in many of its features different from classical counterparts. The reason of these differences is the fact that the convolution operator in (1.1) is bounded in $L^{2}\left(\mathbb{R}^{n}\right)$, and the "potential" is not subordinated to it.

On the other hand, the above operators make sense in a much more general setting, where the convolution is defined on a locally compact noncompact Abelian group $\mathfrak{G}$. Here, in addition to the case where $\mathfrak{G}=\mathbb{R}^{n}$, we have another important example, in which $\mathfrak{G}$ is the field $\mathbb{Q}_{p}$ of p-adic numbers. The spectral theory available in this framework (see the books [1, 6, 7, 21]) deals mostly with perturbations of Vladimirov's fractional differentiation operator, that is does not cover the case of a bounded convolution operator.

In this paper, we concentrate, in the group situation, on the problem of existence of a ground state, the positive eigenfunction corresponding to the maximal positive eigenvalue of $\mathcal{L}$. We show that the approach of [8] based on the use of the Krein-Rutman theorem [12] remains valid in the general group situation. In addition, we derive an existence result from a recurrence property of the random walk corresponding to the density $a$ [14]. For the $p$-adic case, we find explicit conditions for the existence of a ground state, parallel to some results from [8] but possessing certain special features.

\section{Spectral properties. Applications of the Krein-Rutman theorem}

let $\mathfrak{G}$ be a second countable locally compact noncompact Abelian group, $\widehat{\mathfrak{G}}$ be its dual group of continuous characters. The action of a character $z \in \widehat{\mathfrak{G}}$ on an element $x \in \mathfrak{G}$ will be denoted $\langle x, z\rangle$. We write the group operation additively. Let $\mu$ and $\widehat{\mu}$ be the Haar measures on $\mathfrak{G}$ and $\widehat{\mathfrak{G}}$ respectively, normalized in such a way that the Plancherel identity holds, that is the Fourier transform

$$
\tilde{f}(z)=\int_{\mathfrak{G}}\langle x, z\rangle f(x) \mu(d x)
$$

defines an isometric isomorphism from $L^{2}(\mathfrak{G})$ onto $L^{2}(\widehat{\mathfrak{G}})$. The inverse operator is given by the expression

$$
f(x)=\int_{\widehat{\mathfrak{G}}} \overline{\langle x, z\rangle} \tilde{f}(z) \widehat{\mu}(d z) .
$$

See [5, 18, 23] for information regarding harmonic analysis on topological groups. 
Following (1.1) we consider the operator

$$
(\mathcal{L} u)(x)=-m(x) u(x)+\int_{\mathfrak{G}} a(x-y) u(y) \mu(d y)
$$

where $a(x)$ is a nonnegative even function on $\mathfrak{G}$,

$$
\int_{\mathfrak{G}} a(x) \mu(d x)=1,
$$

and the set of zeroes $N(a)=\{x \in \mathfrak{G}: a(x)=0\}$ is empty or compact, $m$ belongs to the Banach space $C_{b}(\mathfrak{G})$ of bounded continuous (in general, complex-valued) functions on $\mathfrak{G}, 0 \leqslant m(x) \leqslant 1$, $m(x) \rightarrow 1$, as $x \rightarrow \infty$ (the notation $x \rightarrow \infty$ means the convergence by the filter generated by complements to compact subsets of $\mathfrak{G})$. It is often convenient to rewrite (2.1) as

$$
\mathcal{L} u=\mathcal{L}_{0} u+V(x) u
$$

where

$$
\left(\mathcal{L}_{0} u\right)(x)=\int_{\mathfrak{G}} a(x-y)[u(y)-u(x)] \mu(d y), \quad V(x)=1-m(x),
$$

so that $0 \leqslant V(x) \leqslant 1$. We assume that $V \in C_{b}(\mathfrak{G})$ and $V(x) \rightarrow 0$, as $x \rightarrow \infty$.

Below we study spectral properties of $\mathcal{L}$ both in $L^{2}(\mathfrak{G})$ and $C_{b}(\mathfrak{G})$.

Lemma 1. Both in $L^{2}(\mathfrak{G})$ and $C_{b}(\mathfrak{G})$, the operator $\mathcal{L}_{0}$ is bounded and dissipative, that is

$$
\left\|\lambda f-\mathcal{L}_{0} f\right\| \geqslant \lambda\|f\| \text { for any } \lambda>0 .
$$

Proof. The case of $L^{2}(\mathfrak{G})$ follows immediately from the representation $\mathcal{L}_{0} f=a * f-f$, properties of the Fourier transform and the fact that in the case of a Hilbert space the condition (2.2) is equivalent to the inequality

$$
\operatorname{Re}\left(\mathcal{L}_{0} x, x\right) \leqslant 0 \text { for all } x \in L^{2}(\mathfrak{G}) .
$$

In the case of $C_{b}(\mathfrak{G})$, the operator $\mathcal{L}_{0}$ is a generator of a strongly continuous contraction semigroup

$$
e^{t \mathcal{L}_{0}}=e^{-t} \sum_{k=0}^{\infty} t^{k} \frac{a^{* k}}{k !}
$$

which implies (2.2).

Note that by (2.3), due to the compactness of $N(a), e^{t \mathcal{L}_{0}}$ is a semigroup of convolution operators with strictly positive kernels. The semigroup $e^{t \mathcal{L}}$ can be given by the Feynman-Kac formula valid for general Markov processes; in the generality sufficient for our situation, that is proved in [4]. By this formula, $e^{t \mathcal{L}}$ has, as well as $e^{t \mathcal{L}_{0}}$, the positivity improving property ([17], Section XIII.12).

It follows from Lemma 1 that the half-plane

$$
\mathcal{D}=\{\lambda \in \mathbb{C}: \operatorname{Re} \lambda>0\}
$$

belongs to the resolvent set of the operator $\mathcal{L}_{0}$. Now we turn to the operator $\mathcal{L}$. 
Lemma 2. The operator $\mathcal{L}$ has only discrete spectrum in the half-plane $\mathcal{D}$.

Proof. For any $\lambda \in \mathcal{D}$ we have

$$
\lambda-\mathcal{L}_{0}-V=\left(\lambda-\mathcal{L}_{0}\right)\left(I-\left(\lambda-\mathcal{L}_{0}\right)^{-1} V\right),
$$

so that

$$
\left(\lambda-\mathcal{L}_{0}-V\right)^{-1}=\left(I-\left(\lambda-\mathcal{L}_{0}\right)^{-1} V\right)^{-1}\left(\lambda-\mathcal{L}_{0}\right)^{-1} .
$$

Writing the Neumann series for $\left(\lambda-\mathcal{L}_{0}\right)^{-1}$ we find that

$$
\left(\lambda-\mathcal{L}_{0}\right)^{-1}=\frac{1}{\lambda+1}+\frac{1}{\lambda+1} A_{\lambda}
$$

where

$$
A_{\lambda}=(\lambda+1)\left(\lambda-\mathcal{L}_{0}\right)^{-1}-1=\sum_{l=1}^{\infty} \frac{a^{* l}}{(\lambda+1)^{l}} .
$$

$A_{\lambda}, \lambda \in \mathcal{D}$, is a bounded convolution operator with an integral kernel

$$
G_{\lambda}(x-y)=\int_{\widehat{\mathfrak{F}}} \overline{\langle x-y, z\rangle} \frac{\tilde{a}(z)}{\lambda+1-\widetilde{a}(z)} \widehat{\mu}(d z),
$$

with

$$
\widetilde{G_{\lambda}}(z)=\frac{\widetilde{a}(z)}{\lambda+1-\widetilde{a}(z)} \in L^{2}(\widehat{\mathfrak{G}}), \quad \lambda \in \mathcal{D} .
$$

It follows from (2.6) that $G_{\lambda} \in L^{1}(\mathfrak{G}) \cap C_{b}(\mathfrak{G})$.

Denote by $W_{\lambda}$ the operator of multiplication by the function

$$
W_{\lambda}(x)=1-\frac{V(x)}{\lambda+1}, \quad \lambda \in \mathcal{D} .
$$

It is a bounded operator with a bounded inverse. It follows from (2.5) that

$$
I-\left(\lambda-\mathcal{L}_{0}\right)^{-1} V=W_{\lambda}-\frac{1}{\lambda+1} A_{\lambda} V,
$$

so that

$$
\left(\lambda-\mathcal{L}_{0}-V\right)^{-1}=\left(I-Q_{\lambda}\right)^{-1}\left(\left(\lambda-\mathcal{L}_{0}\right) W_{\lambda}\right)^{-1}
$$

where

$$
Q_{\lambda}=\frac{1}{\lambda+1} W_{\lambda}^{-1} A_{\lambda} V .
$$

Since $\mathfrak{G}$ is locally compact and second countable, there exists its countable covering by open subsets with compact closures. Taking the subordinate partition of unity, we can form a monotone sequence of continuous nonnegative functions $\left\{h_{r}\right\}$ with compact supports converging pointwise to 1. Let $A_{\lambda}^{(r)}$ be the convolution operator with the convolution kernel $G_{\lambda}^{(r)}(x)=$ $h_{r}(x) G_{\lambda}(x), V^{(r)}$ be the operator of multiplication by $V^{(r)}(x)=h_{r}(x) V(x)$. Then $A_{\lambda}^{(r)}$ is a 
compact operator, and $A_{\lambda}^{(r)} V^{(r)} \rightarrow A_{\lambda} V$ by the norm of operators. Therefore the operator $Q_{\lambda}$ is compact.

Using the analytic Fredholm theorem (see [16, Theorem VI.14, and [19]), we find that $\left(I-Q_{\lambda}\right)^{-1}$ is meromorphic on $\mathcal{D}$. Since $\left(\left(\lambda-\mathcal{L}_{0}\right) W_{\lambda}\right)^{-1}$ is a bounded operator, we conclude that the operator $\mathcal{L}$ has only a discrete spectrum in $\mathcal{D}$.

It follows from (2.6), (2.7) and (2.10) that

(i) $A_{\lambda}$ is a positivity improving operator for each $\lambda>0$, since $G_{\lambda}(x-y)>0, x, y \in \mathfrak{G}$.

(ii) $G_{\lambda}(x-y)$ is monotonically decreasing with respect to $\lambda>0$;

(iii) $Q_{\lambda}, \lambda>0$, is a positivity improving compact integral operator on $C_{b}(\mathfrak{G})$ with the kernel

$$
Q_{\lambda}(x, y)=\frac{G_{\lambda}(x-y) V(y)}{\lambda+1-V(x)}
$$

Let us study the spectral radius $r\left(Q_{\lambda}\right)$ of the operator $Q_{\lambda}, \lambda>0$. Let $\mathcal{E}$ be one of the spaces $C_{b}(\mathfrak{G})$ or $L^{2}(\mathfrak{G})$.

Lemma $3([8])$. The spectral radius $r\left(Q_{\lambda}\right)$ is continuous and monotonically decreasing with respect to $\lambda>0$, and $r\left(Q_{\lambda}\right) \rightarrow 0$ for $\lambda \rightarrow+\infty$. If there exists a function $\varphi \in \mathcal{E}, \varphi \geqslant 0,\|\varphi\|=1$, such that $Q_{\lambda} \varphi(x) \geqslant c_{0} \varphi(x)$, then $r\left(Q_{\lambda}\right) \geqslant c_{0}$.

By (2.4), the equation on the eigenfunction $\psi$,

$$
\left(\mathcal{L}_{0}+V-\lambda\right) \psi=0, \quad \lambda>0,
$$

is equivalent to the equality

$$
Q_{\lambda} \psi=\psi
$$

It follows from Lemma 3 that if

$$
\lim _{\lambda \rightarrow+0} r\left(Q_{\lambda}\right)>1
$$

then there exists such $\lambda>0$ that

$$
r\left(Q_{\lambda}\right)=1, \quad \text { and } r\left(Q_{\lambda^{\prime}}\right)<1 \text { for } \lambda^{\prime}>\lambda .
$$

By the Krein-Rutman theorem ([12, Theorem 6.1), in this case 1 is the maximal positive eigenvalue of $Q_{\lambda}$ with a positive eigenfunction $\psi_{\lambda}>0$. Correspondingly, $\lambda$ is the maximal positive eigenvalue of the operator $\mathcal{L}$, and $\psi_{\lambda}(x)>0$ is the ground state of $\mathcal{L}$. The uniqueness of the ground state follows from the positivity improving property of the semigroup $e^{t \mathcal{L}}$; see Theorem XIII.44 in [17] or Theorem 2.11 in [11].

The following properties of the ground state, provided it exists, are proved just as in [8]. The only difference is that instead of the standard dominated convergence theorem, we use its generalization to the case of convergence with respect to a filter with a countable basis ([2], Chapter 4, §3.7). In our case, this countable basis can be constructed as follows: take the countable increasing sequence of compact sets appearing in the construction of a partition of unity (see e.g. Theorem 5.3 in [13]), and take the sequence of their complements in $\mathfrak{G}$ as the basis of a filter. 
Lemma 4. (i) If the ground state $\psi_{\lambda}$ belongs to $C_{b}(\mathfrak{G})$, then $\psi_{\lambda}(x) \rightarrow 0$, as $x \rightarrow \infty$.

(ii) If the ground state $\psi_{\lambda} \in L^{2}(\mathfrak{G})$, then $\psi_{\lambda} \in C_{b}(\mathfrak{G}) \cap L^{2}(\mathfrak{G})$ and $\psi_{\lambda}(x) \rightarrow 0$, as $x \rightarrow \infty$.

(iii) If the ground state $\psi_{\lambda} \in C_{b}(\mathfrak{G})$ and $V \in L^{2}(\mathfrak{G})$, then $\psi_{\lambda} \in L^{2}(\mathfrak{G})$.

Remark. Let $\mathcal{E}_{1}$ be one of the spaces $C_{b}(\mathfrak{G}), L^{2}(\mathfrak{G})$, and $\mathcal{E}_{2}$ be the other of these spaces. As we see in Lemma 4 , it is a typical situation that a ground state in $\mathcal{E}_{1}$ belongs also to $\mathcal{E}_{2}$. In this case, is it a ground state of the operator in $\mathcal{E}_{2}$ ?

The answer is positive, because in our setting with bounded operators, being an eigenfunction is a pointwise property. By the Krein-Rutman theorem ([12], Theorem 6.1), the operator in $\mathcal{E}_{2}$, having a positive eigenvalue, has also a ground state. In addition, for positivity improving operators, a normalized positive eigenfunction is unique ([1], Theorem 2.11). Therefore the given ground state for $\mathcal{E}_{1}$ is also the ground state for $\mathcal{E}_{2}$.

\section{Random walk}

Let us consider the probability measure $\mu_{a}(d x)=a(x) \mu(d x)$ on the Borel $\sigma$-algebra of the group $\mathfrak{G}$. If $\xi_{1}, \xi_{2}, \ldots, \xi_{m}, \ldots$ are independent $\mathfrak{G}$-valued random variables, each having $\mu_{a}$ for its probability law, then the random walk with an initial point $S_{0}$ is the Markov chain $S_{m}=S_{0}+\xi_{1}+\cdots+\xi_{m}$. Below we connect some properties of the random walk found in [14] with the existence of the ground state of the operator $\mathcal{L}$.

In addition to the classical example of $\mathbb{R}^{n}$ considered in [8], we have the second main example, in which $\mathfrak{G}$ is the additive group of the field $\mathbb{Q}_{p}$ of $p$-adic numbers. Harmonic analysis on $\mathbb{Q}_{p}$ and other local fields is a well-developed branch of contemporary mathematical analysis; see [7, 20, 21].

Recall that $\mathbb{Q}_{p}$ is a completion of the field $\mathbb{Q}$ of rational numbers with respect to the absolute value $|x|_{p}$ defined by setting $|0|_{p}=0$,

$$
|x|_{p}=p^{-\nu} \text { if } x=p^{\nu} \frac{m}{n},
$$

where $\nu, m, n \in \mathbb{Z}$, and $m, n$ are prime to $p \cdot \mathbb{Q}_{p}$ is a locally compact topological field.

Note that by Ostrowski's theorem there are no absolute values on $\mathbb{Q}$, which are not equivalent to the "Euclidean" one, or one of $|\cdot|_{p}$.

The absolute value $|x|_{p}, x \in \mathbb{Q}_{p}$, has the following properties:

$$
\begin{gathered}
|x|_{p}=0 \text { if and only if } x=0 ; \\
|x y|_{p}=|x|_{p} \cdot|y|_{p} \\
|x+y|_{p} \leqslant \max \left(|x|_{p},|y|_{p}\right) .
\end{gathered}
$$

The latter property called the ultra-metric inequality (or the non-Archimedean property) implies the total disconnectedness of $\mathbb{Q}_{p}$ in the topology determined by the metric $|x-y|_{p}$, as well as many unusual geometric properties. Note also the following consequence of the ultra-metric inequality:

$$
|x+y|_{p}=\max \left(|x|_{p},|y|_{p}\right) \quad \text { if }|x|_{p} \neq|y|_{p}
$$


The absolute value $|x|_{p}$ takes the discrete set of non-zero values $p^{N}, N \in \mathbb{Z}$. If $|x|_{p}=p^{N}$, then $x$ admits a (unique) canonical representation

$$
x=p^{-N}\left(x_{0}+x_{1} p+x_{2} p^{2}+\cdots\right),
$$

where $x_{0}, x_{1}, x_{2}, \ldots \in\{0,1, \ldots, p-1\}, x_{0} \neq 0$. The series converges in the topology of $\mathbb{Q}_{p}$. For example,

$$
-1=(p-1)+(p-1) p+(p-1) p^{2}+\cdots, \quad|-1|_{p}=1 .
$$

We denote $\mathbb{Z}_{p}=\left\{x \in \mathbb{Q}_{p}:|x|_{p} \leqslant 1\right\}$. $\mathbb{Z}_{p}$, as well as all balls in $\mathbb{Q}_{p}$, is simultaneously open and closed.

Proceeding from the canonical representation (3.1) of an element $x \in \mathbb{Q}_{p}$, we define the fractional part of $x$ as the rational number

$$
\{x\}_{p}= \begin{cases}0, & \text { if } N \leqslant 0 \text { or } x=0 ; \\ p^{-N}\left(x_{0}+x_{1} p+\cdots+x_{N-1} p^{N-1}\right), & \text { if } N>0 .\end{cases}
$$

The function $\chi(x)=\exp \left(2 \pi i\{x\}_{p}\right)$ is an additive character of the field $\mathbb{Q}_{p}$, that is a character of its additive group. It is clear that $\chi(x)=1$ if $|x|_{p} \leqslant 1$. Denote by $d x$ the Haar measure on the additive group of $\mathbb{Q}_{p}$ normalized by the equality $\int_{\mathbb{Z}_{p}} d x=1$. The above additive group is self-dual - every continuous character can be written as $\langle x, z\rangle=\chi(z x), x \in \mathbb{Q}_{p}$, where $z \in \mathbb{Q}_{p}$ is a unique element defining the character.

Returning to random walk on a general group $\mathfrak{G}$ we describe the assumption from [14], under which the result we need was obtained.

$\left(\mathfrak{G}_{0}\right)$ : The minimal closed subgroup of $\mathfrak{G}$ generated by $\operatorname{supp} \mu_{a}$ coincides with $\mathfrak{G}$.

If $\widetilde{a}(z)=1$ where $z \neq 0$ (note that 0 is the unit character; this additive notation corresponds, for example, to the cases of $\mathbb{R}$ or $\left.\mathbb{Q}_{p}\right)$, then

$$
1-\widetilde{a}(z)=\int_{\mathfrak{G}}(1-\langle x, z\rangle) a(x) \mu(d x),
$$

so that $\langle x, z\rangle=1$ for $x \in \operatorname{supp} \mu_{a}$, thus for all $x$ from the subgroup generated by $\operatorname{supp} \mu_{a}$, so that due to $\left(\mathfrak{G}_{0}\right),\langle x, z\rangle=1$ for all $x \in \mathfrak{G}$. This contradiction proves that

$$
\widetilde{a}(z) \neq 1 \text { for } z \neq 0 .
$$

For $\mathfrak{G}=\mathbb{R}\left(\right.$ or $\left.\mathbb{R}^{n}\right)$, the measures whose Fourier transforms equal 1 at nonzero points (or slightly more general ones) are called the lattice distributions and are concentrated on lattices (see Section 1.5 in [15] or Section XV.1 in [3]). This is of course impossible in the case of a continuous density.

For the case of $\mathbb{Q}_{p}$ (or, similarly, for $\mathbb{Q}_{p}^{n}$ ) suppose that supp $a \subset\left\{x \in \mathbb{Q}_{p}:|x|_{p} \leqslant p^{N}\right\}$, $N \in \mathbb{Z}$. We have

$$
1-\widetilde{a}(\xi)=\int_{|x|_{p} \leqslant p^{N}}\left[1-\cos \left(2 \pi\{x \xi\}_{p}\right)\right] a(x) d x,
$$


so that $1-\widetilde{a}(\xi)=0$ for $|\xi| \leqslant p^{-N}$. Thus, in the $p$-adic case, the property (3.2) is violated, if $a$ has a compact support. Conversely, if $\widetilde{a}(\xi)=1$ for $\xi \neq 0$, then $a(x)=0$ for $|x|_{p}>|\xi|_{p}^{-1}$. On the other hand, this case is excluded by the condition $\left(\mathfrak{G}_{0}\right)$ - by the ultrametric inequality, a $p$-adic ball does not generate the additive group of $\mathbb{Q}_{p}$.

A random walk is said to be recurrent, if for some compact neighborhood $M$ of 0 ,

$$
\sum_{m=1}^{\infty} \mathbf{P}\left\{S_{m} \in M \mid S_{0}=0\right\}=\infty .
$$

Theorem 1. Suppose that $\mathfrak{G}$ is a noncompact second countable locally compact Abelian group, and $a(x)$, in addition to the assumptions made in Introduction, is such that $\left(\mathfrak{G}_{0}\right)$ is satisfied, and the corresponding random walk is recurrent. Then for any $V \neq \equiv 0$ satisfying the conditions from Section 2, the ground state of the operator $\mathcal{L}$ in $C_{b}(\mathfrak{G})$ exists.

Proof. It follows from the recurrence of our random walk that

$$
\int_{P} \frac{1}{1-\widetilde{a}(z)} \widehat{\mu}(d z)=\infty
$$

for any open neighborhood $P$ of the origin in $\widehat{\mathfrak{G}}$ ([14], Theorem 5.1).

Let us take a continuous real-valued function $\varphi$ with a compact support, $\|\varphi\|=1$, such that

$$
\widetilde{V \varphi}(0)=\int_{\mathfrak{G}} V(x) \varphi(x) \mu(d x)>0 .
$$

The assumptions regarding $a(x)$ and $V(x)$ imply that $\widetilde{a}, \widetilde{V} \varphi \in L^{2}(\mathfrak{G}) \cap C_{b}(\mathfrak{G})$ and consequently, $\widetilde{a} \widetilde{V} \varphi \in L^{1}(\mathfrak{G}) \cap C_{b}(\mathfrak{G})$. Note also that $\widetilde{a}(z) \rightarrow 0$, as $z \rightarrow \infty$ ([18], Theorem 1.2.4). Then by (2.7), for any $x \in \operatorname{supp} \varphi$,

$$
\begin{aligned}
& \left(Q_{\lambda} \varphi\right)(x)=\frac{1}{\lambda+1-V(x)} \int_{\mathfrak{G}} G_{\lambda}(x-y) V(y) \varphi(y) \mu(d y) \\
& =\frac{1}{\lambda+1-V(x)} \int_{\mathfrak{G}} V(y) \varphi(y) \mu(d y) \int_{\widehat{\mathfrak{G}}}\langle x-y, z\rangle \frac{\widetilde{a}(z)}{\lambda+1-\widetilde{a}(z)} \widehat{\mu}(d z) \\
& \quad=\frac{1}{\lambda+1-V(x)} \int_{\widehat{\mathfrak{G}}} \frac{\langle-x, z\rangle \widetilde{a}(z) \widetilde{V} \varphi(z)}{\lambda+1-\widetilde{a}(z)} \widehat{\mu}(d z) .
\end{aligned}
$$

Since all functions appearing in the above expression of $Q_{\lambda} \varphi$ as an integral on $\mathfrak{G}$ are realvalued, we may write

$$
\left(Q_{\lambda} \varphi\right)(x)=\frac{1}{\lambda+1-V(x)} \int_{\widehat{\mathfrak{G}}} \frac{\widetilde{a}(z) \operatorname{Re}\{\langle-x, z\rangle \widetilde{V \varphi}(z)\}}{\lambda+1-\widetilde{a}(z)} \widehat{\mu}(d z) .
$$


Choose such a neighborhood $\mathfrak{P}$ of the origin in $\widehat{\mathfrak{G}}$ that

$$
\widetilde{a}(z) \operatorname{Re}\{\langle-x, z\rangle \widetilde{V \varphi}(z)\} \geqslant \gamma_{0}>0, \quad z \in \mathfrak{P}
$$

and write the integral in (3.4) as the sum of integrals over $\mathfrak{P}$ and $\widehat{\mathfrak{G}} \backslash \mathfrak{P}$. Due to (3.2), the second of them has a finite limit, as $\lambda \rightarrow 0$. By the monotone convergence theorem, it follows from (3.3) and (3.4) that the integral over $\mathfrak{P}$ tends to $+\infty$, as $\lambda \rightarrow+0$.

The continuous function $U_{\lambda}(x)=\left[\left(Q_{\lambda} \varphi\right)(x)\right]^{-1}$ tends to 0 monotonically, as $\lambda \rightarrow 0$ (this monotonicity follows from the monotone dependence of $G_{\lambda}$ on $\lambda$ ). By the Dini theorem, this convergence is uniform on $\operatorname{supp} \varphi$. Therefore for any $c_{0}>0$, there exists such $\lambda>0$ that $\left(Q_{\lambda} \varphi(x) \geqslant c_{0} \varphi(x)\right.$. By Lemma 3, this means that $\lim _{\lambda \rightarrow+0} r\left(Q_{\lambda}\right)=\infty$, which implies the inequality (2.13) and the existence of a ground state.

\section{The $p$-adic case}

In this section we give explicit conditions for the existence of a ground state in the case where $G=\mathbb{Q}_{p}^{n}$. We consider mostly the case of the ground state from $C_{b}\left(\mathbb{Q}_{p}^{n}\right)$. The existence of a ground state for operators on $L^{2}\left(\mathbb{Q}_{p}^{n}\right)$ can be proved just as it is done for operators on $L^{2}\left(\mathbb{R}^{n}\right)$ in $([8]$, Section 4).

Let us introduce some notation for $\mathbb{Q}_{p}^{n}=\underbrace{\mathbb{Q}_{p} \times \cdots \times \mathbb{Q}_{p}}_{n \text { times }}$. This is a vector space over the field $\mathbb{Q}_{p}$ with the norm

$$
\|x\|=\max _{1 \leqslant j \leqslant n}\left|x_{j}\right|_{p}, \quad x=\left(x_{1}, \ldots, x_{n}\right) \in \mathbb{Q}_{p}^{n} .
$$

$B_{N}(N \in \mathbb{Z})$ will denote the ball $\left\{x \in \mathbb{Q}_{p}^{n}:\|x\| \leqslant p^{N}\right\}$. Just as for $\mathbb{Q}_{p}$, the additive group of $\mathbb{Q}_{p}^{n}$ is self-dual. The simplest integration formulas are

$$
\int_{\|x\| \leqslant p^{j}} d^{n} x=p^{n j}, \quad \int_{\|x\|=p^{j}} d^{n} x=\left(1-p^{-n}\right) p^{n j}, \quad j \in \mathbb{Z}
$$

(see e.g. [22]). The Fourier transform of a function $f: \mathbb{Q}_{p}^{n} \rightarrow \mathbb{C}$ is defined as

$$
\tilde{f}(\xi)=\int_{\mathbb{Q}_{p}^{n}} \chi(x \cdot \xi) f(x) d^{n} x
$$

where $x \cdot \xi=\sum_{j=1}^{n} x_{j} \xi_{j}$. As before, the Fourier transform of an even function, for example of $a$, is real-valued.

The next result gives a simple example of the existence of a ground state.

Theorem 2. Suppose that $V(x)=1$ for $x \in B_{N}, N \in \mathbb{Z}$. Then the ground state of $\mathcal{L}$ exists. 
Proof. Let $f_{N}$ be the indicator of the ball $B_{N}$. Since $B_{N}$ is simultaneously open and closed, $f_{N} \in C_{b}\left(\mathbb{Q}_{p}^{n}\right)$. For any $\lambda \in(0,1)$ and any $x \in B_{N}$, we get

$$
\left(Q_{\lambda} f_{N}\right)(x)=\int_{B_{N}} Q_{\lambda}(x, y) d y \geqslant \frac{1}{\lambda} \int_{B_{N}} G_{\lambda}(x-y) d y \geqslant \frac{\operatorname{Vol}\left(B_{N}\right)}{\lambda} \varkappa
$$

where $\varkappa=\min _{x, y \in B_{N}} G_{1}(x-y)<\min _{\lambda \in(0,1)} \min _{x, y \in B_{N}} G_{\lambda}(x-y)$.

By Lemma 3, $\lim _{\lambda \rightarrow+0} r\left(Q_{\lambda}\right)=\infty$, which implies the existence of a ground state.

In our next result, we begin with a ground state in $L^{2}\left(\mathbb{Q}_{p}^{n}\right)$, and then use Remark after Lemma 4.

Theorem 3. Assume that for some $\beta \in(0,1)$, there exists such $N \in \mathbb{Z}$ that

$$
\beta \leqslant V(x) \leqslant 1, \quad x \in B_{N} .
$$

Then the ground state of the operator $\mathcal{L}$ exists, if $N=N(\beta)$ is sufficiently large.

Proof. To prove the existence of a ground state $\psi_{\lambda} \in L^{2}\left(\mathbb{Q}_{p}^{n}\right)$, it suffices to show that the quadratic form $(\mathcal{L} f, f)$ is positive for some $f \in L^{2}\left(\mathbb{Q}_{p}^{n}\right)$. Taking the indicator $f_{N}$, as in the proof of Theorem 2, we have

$$
\left(\mathcal{L} f_{N}, f_{N}\right)=\left(\mathcal{L}_{0} f_{N}, f_{N}\right)+\left(V f_{N}, f_{N}\right)
$$

and

$$
\left(V f_{N}, f_{N}\right) \geqslant \beta \operatorname{Vol}\left(B_{N}\right)
$$

Next, if $\|x\| \leqslant p^{N}$, then

$$
\left(\mathcal{L}_{0} f_{N}\right)(x)=-\int_{\|y\|>p^{N}} a(x-y) d y
$$

so that

$$
-\left(\mathcal{L}_{0} f_{N}, f_{N}\right)=\int_{\|x\| \leqslant p^{N}} d x \int_{\|y\|>p^{N}} a(x-y) d y .
$$

The change of variables $y=x-z$ implies (note that $\|z\|=\|y\|$ ) the equality

$$
-\left(\mathcal{L}_{0} f_{N}, f_{N}\right)=\int_{\|x\| \leqslant p^{N}} d x \int_{\|z\|>p^{N}} a(z) d z
$$

and the relation

$$
\frac{1}{\operatorname{Vol}\left(B_{N}\right)}\left(\mathcal{L}_{0} f_{N}, f_{N}\right) \rightarrow 0, \quad \text { as } N \rightarrow \infty .
$$

Comparing (4.1) and (4.2) we find that $\mathcal{L}$ has a positive discrete spectrum, hence a ground state, if $N$ is large enough. 
By our assumption, $N(a)$ is empty or compact. Hence the function $a$ cannot have a compact support, so that $\widetilde{a}(\xi) \neq 1$ for $\xi \neq 0$. Let us study the behavior of $\widetilde{a}(\xi)$ near the origin.

Denote

$$
A_{j}=\sup _{\|x\|=p^{j}} a(x), \quad j \in \mathbb{Z}
$$

Lemma 5. If for some $l \geqslant 0$,

$$
\sum_{N=l}^{\infty} \frac{1}{p^{N n} \sum_{j=N+1}^{\infty} p^{j n} A_{j}}=\infty
$$

then

$$
\int_{\|\xi\| \leqslant p^{-l}} \frac{d^{n} \xi}{1-\widetilde{a}(\xi)}=\infty
$$

Proof. Since the function $a$ is even, $\widetilde{a}$ is real-valued. We have

$$
\begin{array}{r}
1-\tilde{a}(\xi)=\int_{Q p n}[1-\chi(\xi \cdot x)] a(x) d^{n} x=\sum_{j=-\infty}^{\infty} \int_{\|x\|=p^{j}}[1-\operatorname{Re} \chi(\xi \cdot x)] a(x) d^{n} x \\
\leqslant \sum_{j=-\infty}^{\infty} A_{j} \int_{\|x\|=p^{j}}[1-\operatorname{Re} \chi(\xi \cdot x)] d^{n} x .
\end{array}
$$

The integral $\int_{\|x\|=p^{j}} \operatorname{Re} \chi(\xi \cdot x) d^{n} x=\int_{\|x\|=p^{j}} \chi(\xi \cdot x) d^{n} x$ is given in [22] (formula (15.10):

$$
\int_{\|x\|=p^{j}} \chi(\xi \cdot x) d^{n} x=\left(1-p^{-n}\right) p^{j n} \cdot\left\{\begin{array}{ll}
1, & \text { if }\|\xi\| \leqslant p^{-j} \\
0, & \text { otherwise }
\end{array} \quad-p^{(j-1) n} \cdot \begin{cases}1, & \text { if }\|\xi\|=p^{-j+1} \\
0, & \text { otherwise. }\end{cases}\right.
$$

Let $\|\xi\|=p^{-N}, N \geqslant 0$. If $N \geqslant j$, then

$$
\int_{\|x\|=p^{j}} \chi(\xi \cdot x) d^{n} x=\left(1-p^{-n}\right) p^{j n}
$$

If $N=j-1$, then

$$
\int_{\|x\|=p^{j}} \chi(\xi \cdot x) d^{n} x=-p^{(j-1) n}=-p^{N n}
$$

For other values of $j$, the integral equals 0 . Therefore

$$
\int_{\|x\|=p^{j}}[1-\chi(\xi \cdot x)] d^{n} x= \begin{cases}0, & \text { if } j \leqslant N \\ \left(1-p^{-n}\right) p^{j n}+p^{N n}=p^{(N+1) n}, & \text { if } j=N+1 \\ \left(1-p^{-n}\right) p^{j n}, & \text { if } j \geqslant N+2\end{cases}
$$


and we obtain the inequality

$$
0 \leqslant 1-\widetilde{a}(\xi) \leqslant \sum_{j=N+1}^{\infty} p^{j n} A_{j}
$$

hence the inequality

$$
\int_{\|\xi\| \leqslant p^{-l}} \frac{d^{n} \xi}{1-\widetilde{a}(\xi)} \geqslant\left(1-p^{-n}\right) \sum_{N=l}^{\infty} \frac{1}{p^{N n} \sum_{j=N+1}^{\infty} p^{j n} A_{j}}
$$

Now the condition (4.3) implies (4.4).

The next theorem follows from Lemma 5, just as Theorem 1 was deduced from a result obtained in [14].

Theorem 4. For any functions $a$ and $V \not \equiv 0$ satisfying the assumptions from Introduction and Section 2, as well as the condition (4.3), a ground state from $C_{b}\left(\mathbb{Q}_{p}^{n}\right)$ exists.

Remark. If the function $a$ is radial, that is $a(x)=\mathcal{A}(\|x\|)$, then $A_{j}=\mathcal{A}\left(p^{j}\right)$ and

$$
\sum_{j=N+1}^{\infty} p^{j n} A_{j}=\left(1-p^{-n}\right)^{-1} \int_{\|x\| \geqslant p^{N+1}} a(x) d^{n} x .
$$

\section{Acknowledgments}

The first-named author is grateful to the Bielefeld University for hospitality during his visits to Bielefeld. The work of the first author was also supported in part by Grant 23/16-18 "Statistical dynamics, generalized Fokker-Planck equations, and their applications in the theory of complex systems" of the Ministry of Education and Science of Ukraine. The second-named author gratefully acknowledges the financial support by the DFG through CRC 701 Stochastic Dynamics: Mathematical Theory and Applications and the European Commission under the project STREVCOMS PIRSES-2013- 612669.

\section{References}

[1] S. Albeverio, A. Yu. Khrennikov and V. M. Shelkovich, Theory of p-Adic Distributions. Linear and Nonlinear Models. Cambridge University Press, 2010.

[2] N. Bourbaki, Elements of Mathematics. Integration I, Springer, Berlin, 2004.

[3] W. Feller, An Introduction to Probability Theory and Its Applications, Vol. 2, Wiley, New York, 1971. 
[4] R. K. Getoor, Additive functionals of a Markov process, Pacif. J. Math. 7 (1957), 15771591.

[5] E. Hewitt and K. A. Ross, Abstract Harmonic Analysis, Vols. I, II, Springer, Berlin, 1963, 1970.

[6] A. Yu. Khrennikov, S. V. Kozyrev and W. Zúñiga-Galindo, Ultrametric PseudoDifferential Equations and Applications, Cambridge University Press, 2018.

[7] A. N. Kochubei, Pseudo-Differential Equations and Stochastics over Non-Archimedean Fields, Marcel Dekker, New York, 2001.

[8] Yu. Kondratiev, S. Molchanov, S. Pirogov and E. Zhizhina, On ground state of some non local Schrodinger operators, Appl. Anal. 96 (2017), 1390-1400.

[9] Yu. Kondratiev, S. Molchanov, A. Piatnitski and E. Zhizhina, Resolvent bounds for jump generators. Appl. Anal. 97 (2018), 323-336.

[10] Yu. Kondratiev, S. Molchanov and B. Vainberg, Spectral analysis of non-local Schrodinger operators, J. Funct. Anal. 273 (2017), 1020-1048.

[11] M. A. Krasnosel'skii, Positive Solutions of Operator Equations, Noordhoff, Groningen, 1964.

[12] M. G. Krein and M. A. Rutman, Linear operators leaving invariant a cone in a Banach space, Amer. Math. Soc. Translation, no. 26 (1950), 128 pp.

[13] S. Lang, Real and Functional Analysis, Springer, New York, 1993.

[14] S. C. Port and C. J. Stone, Potential theory of random walks on Abelian groups, Acta Math. 122 (1969), 19-114.

[15] B. Ramachandran, Advanced Theory of Characteristic Functions, Statistical Publishing Society, Calcutta, 1967.

[16] M. Reed and B. Simon, Methods of Modern Mathematical Physics, Vol. 1, Academic Press, New York, 1972.

[17] M. Reed and B. Simon, Methods of Modern Mathematical Physics, Vol. 4, Academic Press, New York, 1978.

[18] W. Rudin, Fourier Analysis on Groups, Interscience, New York, 1962.

[19] S. Steinberg, Meromorphic families of compact operators, Arch. Rat. Mech. Anal. 31 (1968), 372-379.

[20] M. H. Taibleson, Fourier Analysis on Local Fields, Princeton University Press, 1975.

[21] V. S. Vladimirov, I. V. Volovich and E. I. Zelenov, p-Adic Analysis and Mathematical Physics, World Scientific, Singapore, 1994. 
[22] V. S. Vladimirov, Tables of Integrals of Complex-Valued Functions of p-Adic Arguments, Steklov Mathematical Institute, Moscow, 2003 (Russian). English version, ArXiv: math-ph/9911027.

[23] A. Weil, L'Intégration dans les Groupes Topologiques et ses Applications, Hermann, Paris, 1965. 